\title{
Unusual cystic presentation of pulmonary nodular amyloidosis associated with
} MALT-type lymphoma

\author{
S. Lantuejoul*, N. Moulai*, S. Quetant" , P.Y. Brichon`, C. Brambilla\#, \\ E. Brambilla* and G.R. Ferretti ${ }^{+}$
}

\begin{abstract}
The case reported herein consists of nodular pulmonary amyloidosis presenting with unusual cystic radiological features which reveal a pulmonary localisation of an extranodal marginal zone B-cell lymphoma of mucosa-associated lymphoid tissue (MALT lymphoma).

The present case is the first to report a radiological presentation of nodular pulmonary amyloidosis in the absence of Sjögren's syndrome.

Although transthoracic fine-needle biopsy was helpful for the diagnostic of amyloidosis, final diagnosis of associated MALT-type lymphoma required an open lung biopsy.

This emphasises the importance of performing surgical investigations in pulmonary nodular amyloidosis in order to depict the presence of underlying lung tumours or lymphoproliferative disorders.
\end{abstract}

KEYWORDS: Amyloidosis, cystic, lung, lymphoma, mucosa-associated lymphoid tissue, nodular

$\Delta$ ssociation of pulmonary amyloidosis with lymphoma is a classical albeit rare finding [1-4], reported mainly with extranodal marginal zone B-cell lymphoma of mucosaassociated lymphoid tissue (MALT lymphoma) of gastrointestinal $[5,6]$ or pulmonary origin $[1$, 2]. Pulmonary nodular amyloidosis is a localised form of pulmonary amyloidosis, in addition to tracheobronchial and diffuse alveolo-septal amyloidosis, characterised histologically by extracellular deposits of amyloid-type AL protein, homologous to the variable portion of immunoglobulin light chains, predominantly of $\lambda$ type. Pulmonary nodular amyloidosis mainly occurs in elderly patients who harbour an indolent clinical presentation; incidentally, nodules are most frequently found on chest radiographs or at post mortem examination. Prognosis is usually excellent, although nodular pulmonary amyloidosis can obscure underlying lymphoproliferative disorders, including lymphoma. Whereas pulmonary amyloid deposit demonstrated using fineneedle biopsy [7], the present study reports a case of nodular pulmonary amyloidosis presenting with unusual cystic features on the computed tomography (CT) scan, which, in relation to a pulmonary localisation of MALT-type lymphoma, could only be demonstrated by surgical lung biopsy.

\section{CASE HISTORY}

A 50-yr-old female ex-smoker (20 pack-yrs) was referred to the present authors' institution (Dept of Pathology, CHU A Michallon, Grenoble, France) in April 2004 for multiple pulmonary nodules discovered on a systematic chest radiograph. Past history included epigastralgias 1 month previously, with gastric biopsies favouring a gastric MALT-type lymphoma related to Helicobacter pylori. A bone marrow biopsy performed as a result showed bone marrow involvement by the same lymphoid proliferation and confirmed its $\lambda$ monoclonality. A high-resolution CT scan 1 month later revealed eight ill-defined nodules of different sizes associated with cysts, and distributed through both lungs. Some of them were calcified and bronchiolocentric (fig. 1). A fine-needle transthoracic biopsy was performed and showed amorphous eosinophilic material suggestive of amyloidosis engulfed by giant cells and surrounded by a few crushed lymphocytes. This amorphous material was revealed by thioflavine $\mathrm{T}$ staining under UV light, but remained negative with anti-serum
AFFILIATIONS

Depts of *Pathology,

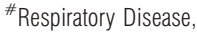

"Cardiovascular and Thoracic

Surgery, and

+Radiology, INSERM U578 CHU A

Michallon, Grenoble, France.

CORRESPONDENCE

E. Brambilla

Dept of Pathology

CHU A Michallon

BP 217

38043 Grenoble Cedex 09

France

Fax: 33476765949

E-mail: EBrambilla@chu-grenoble.fr

Received:

November 202005

Accepted after revision:

January 162007

STATEMENT OF INTEREST

None declared. 


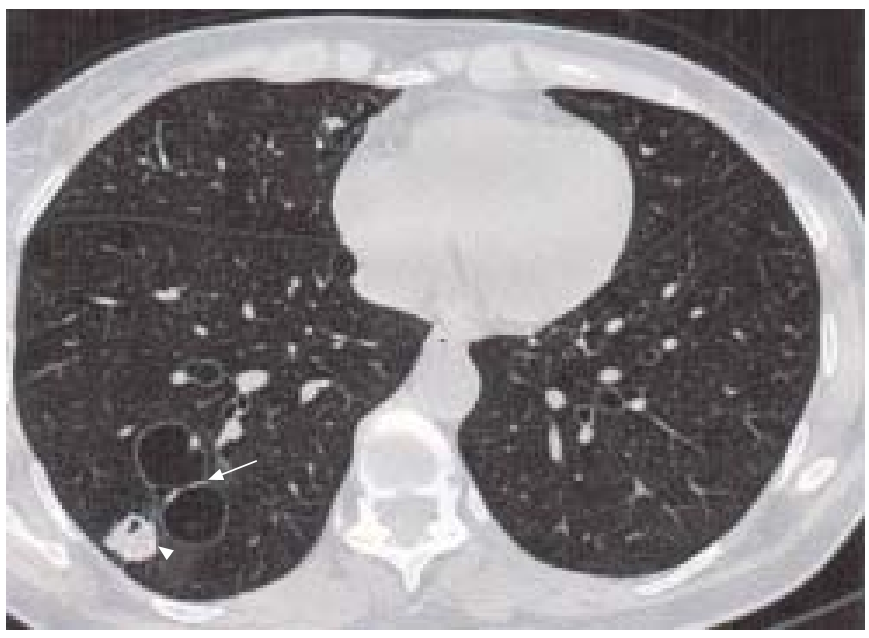

FIGURE 1. High-resolution computed tomography scan (1 mm slice thickness) at the level of the heart shows thin-walled cysts (arrow) within the right lung and one pulmonary nodule of soft tissue density that contains eccentric nodular calcifications (arrowhead)

amyloid A (SAA) protein immunostaining. No evidence of lymphoid proliferation was noticed.

Two partially cavitated nodules were surgically resected because the high suspicion of a pulmonary localisation of lymphoma requires a more aggressive treatment. At histology, the nodules were widely composed of amyloid deposits, with foci of calcification and ossification (fig. 2). Amyloid deposits were again positively stained with thioflavine T (fig. 3) and remained negative with anti-SAA antibody. They were surrounded by sheets of small plasmacytoid lymphocytes admixed with large lymphocytes expressing CD20, but not CD5, CD10, CD43 and CD23 antigens at immunohistochemistry (fig. 4). These lymphocytes focally invaded the pleura and surrounded bronchovascular bundles, infiltrating bronchiolar epithelium to form typical lymphoepithelial lesions (fig. 5).
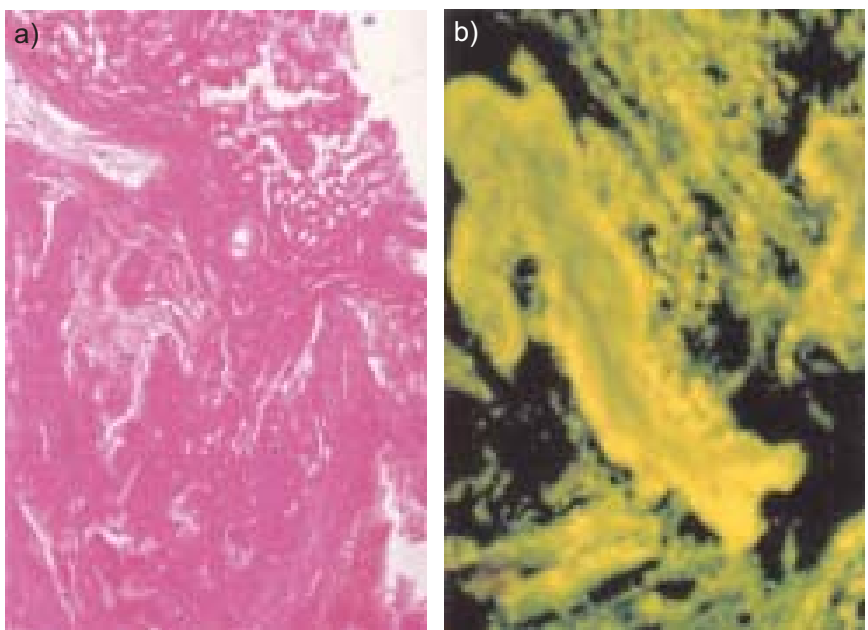

FIGURE 3. a) Amyloid deposit at high magnification consisting of a solid mass of amorphous eosinophilic extracellular material (haematoxylin-eosin stain). b) Amyloid deposits strongly fluorescing with thioflavine T stain under UV light.

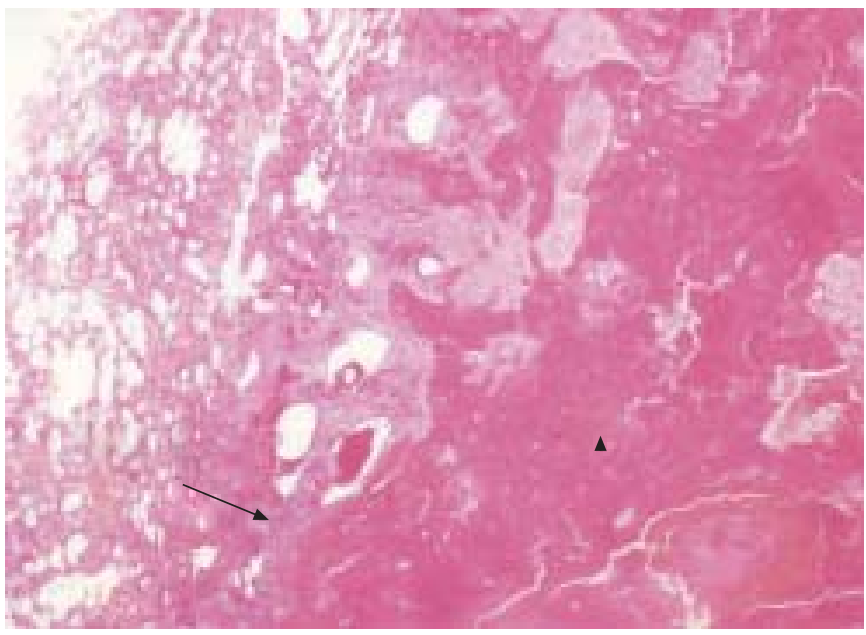

FIGURE 2. Histological examination of one nodule, mainly composed of amyloid deposits (arrowhead), surrounded by a lymphoplasmacytoid proliferation extending to the adjacent normal lung (arrow; haematoxylin-eosin-saffron stain)

Cystic lesions corresponded to dilated bronchioles infiltrated by amyloidosis (fig. 6). Overall, the diagnosis of a stage IV marginal zone lymphoma of MALT-type with gastric, bone marrow and pulmonary involvement was established and the patient was treated by rituximab chemotherapy and bone marrow transplantation. The patient is alive and well 17 months on, with no modification of the volume of the nodules and the cysts on the radiological controls.

\section{DISCUSSION}

The present authors report a case of a pulmonary localisation of MALT-type lymphoma associated with pulmonary nodular amyloidosis presenting with unusual findings on the CT scan. Radiological patterns of pulmonary MALT-type lymphoma are numerous, often found in combination, and consist of localised alveolar opacities, nodules and/or interstitial infiltrates. In
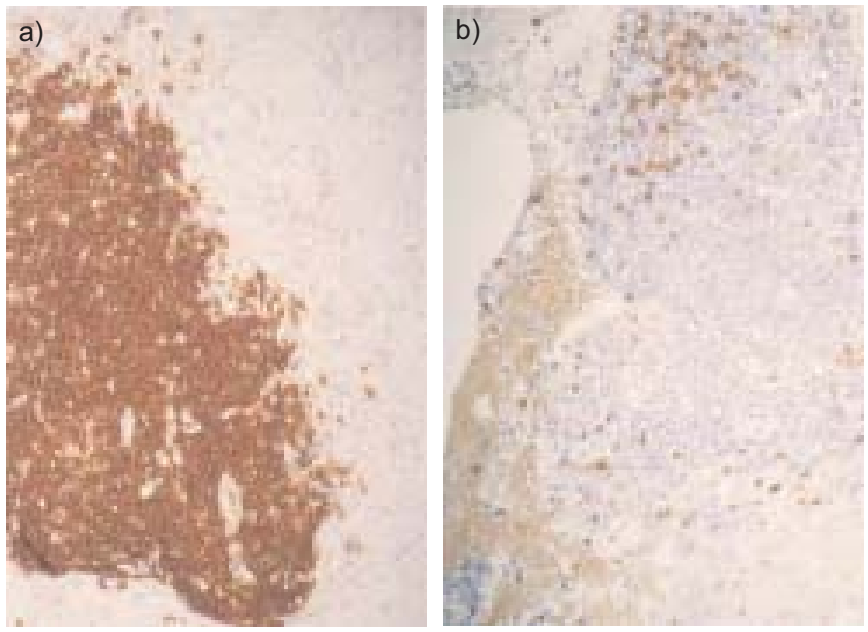

FIGURE 4. Strong expression of the lymphoid proliferation of (a) CD20, whereas only T-cell lymphocytes, and no malignant cells, expressed CD5 (b; immunoperoxidase stain) 


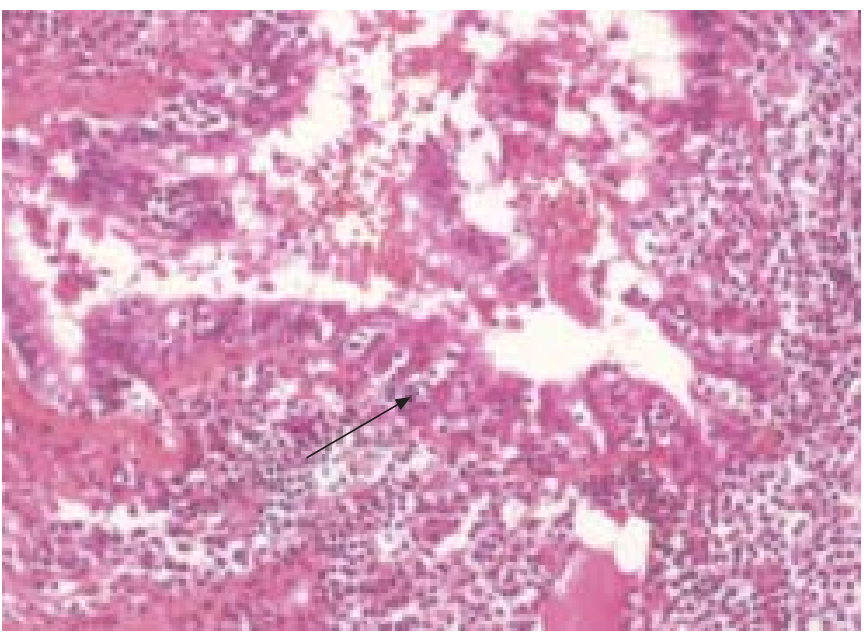

FIGURE 5. At higher magnification, small lymphoplasmacytoid lymphocytes infiltrate the bronchiolar epithelium, forming classical lymphoepithelial lesions (arrow; haematoxylin-eosin-saffron stain)

contrast, pulmonary nodular amyloidosis predominantly presents with tumour-like nodules, bilateral but asymmetrical in distribution, and is often located in the lower lobes. They range in size from $0.4-5 \mathrm{~cm}$ [8], and they classically contain calcification or bone formation. Very occasionally, pulmonary nodular amyloidosis may present with bronchiolocentric nodules abutting large thin-walled cysts with internal septae. These findings have been described in patients with Sjögren's syndrome, either with or without lymphoid interstitial pneumonia $[9,10]$ or in association with lymphoproliferative disease [11]. One case of nodular amyloidosis with bullae has also been observed without any underlying inflammatory or proliferative disorder [12], and one case was reported in association with alveoloseptal amyloidosis [13]. The present case is the first to document cystic pulmonary nodular amyloidosis related to MALT-type lymphoma in the absence of Sjögren's syndrome, as the patient did not present any of the revised diagnostic criteria from the American-European Consensus Group for this disease [14].

As the authors' first diagnosis based on fine-needle biopsy was restricted to pulmonary nodular amyloidosis, it must be kept in mind that the amyloidosis may be isolated or may accompany either benign or malignant lymphoproliferations, including nodular lymphoid hyperplasia, lymphoid interstitial pneumonia and lymphoma, in addition to connective tissue diseases and HIV infection [1, 4]. Other malignant lung tumours can also exhibit amyloid deposits, such as carcinoids, which may contain an amyloid stroma, which is likely to be composed of calcitonin-related gene peptide or to be neuropeptide derived [15], or squamous cell and bronchioloalveolar carcinomas, where amyloid deposits are more related to systemic amyloidosis [16, 17]. In addition, metastatic renal cell carcinoma may exhibit an SAA-rich stroma [18], and pulmonary localisation of light-chain disease can masquerade as amyloidosis by tissue deposition of nonamyloid $\kappa$ chains [19].

The mechanism of cyst formation in pulmonary nodular amyloidosis remains unknown, but is thought to be due to a ball-valve mechanism, responsible for air trapping within

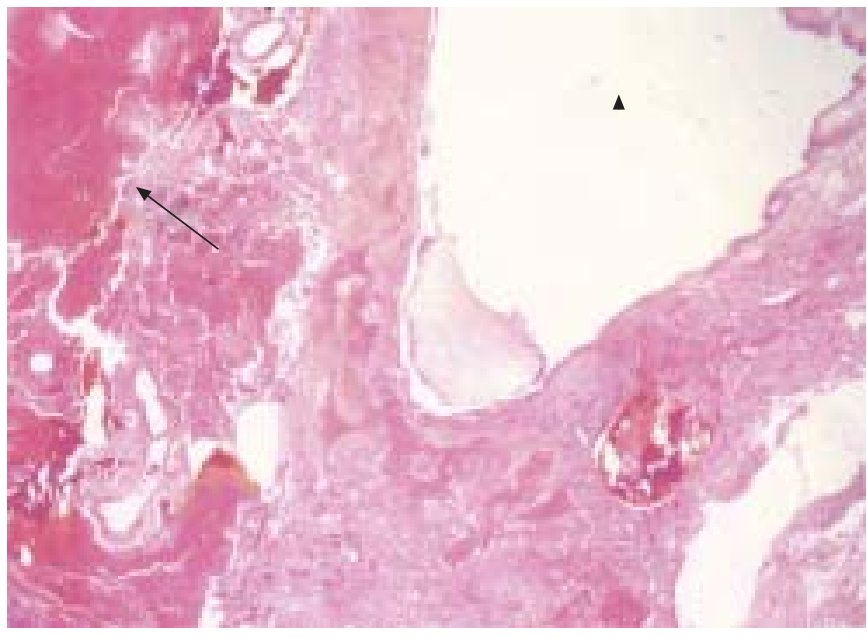

FIGURE 6. Cystic lesions formed by dilated bronchioles (arrowhead) infiltrated by amyloid deposits (arrow).

narrowed airways infiltrated either by amyloid deposits and/ or malignant lymphoid proliferation, as in the present case. Another hypothesis is that amyloid deposits within bronchioloalveolar structures and around alveolar capillaries could favour an ischaemic process leading to disruption of alveolar walls [13].

In conclusion, the present authors report a case of pulmonary localisation of mucosa-associated lymphoid tissue-type lymphoma associated with pulmonary nodular amyloidosis presenting with unusual cystic features on computed tomography. The present case study illustrates the fact that, although a fine-needle biopsy may be sufficient for the diagnosis of pulmonary nodular amyloidosis, the presence of an associated tumour must be considered in the differential diagnosis of amyloid deposits and may justify additional surgical investigations for diagnosis and staging.

\section{REFERENCES}

1 Lim JK, Kurtin PJ, Kyle RA, Gertz MA. Pulmonary marginal zone lymphoma of MALT type as a cause of localized pulmonary amyloidosis. J Clin Pathol 2001; 54: 642-646.

2 Davis CJ, Butchart EG, Gibbs AR. Nodular pulmonary amyloidosis occurring in association with pulmonary lymphoma. Thorax 1991; 46: 217-218.

3 Glenner GG. Amyloid deposits and amyloidosis. The betafibrilloses. N Engl J Med 1980; 302: 1283-1292, 1333-1343.

4 Azzopardi JG, Lehner T. Systemic amyloidosis and malignant disease. J Clin Pathol 1966; 19: 539-548.

5 Goteri G, Ranaldi R, Pileri SA, Bearzi I. Localized amyloidosis and gastrointestinal lymphoma: a rare association. Histopathology 1998; 32: 348-355.

6 Caulet S, Robert I, Bardaxoglou E, et al. Malignant lymphoma of mucosa associated lymphoid tissue: a new etiology of amyloidosis. Pathol Res Pract 1995; 191: 1203-1207.

7 Mollers MJ, van Schaik JP, van der Putte SC. Pulmonary amyloidoma. Histologic proof yielded by transthoracic coaxial fine needle biopsy. Chest 1992; 102: 1597-1598. 
8 Utz JP, Swensen SJ, Gertz MA. Pulmonary amyloidosis. The Mayo clinic experience from 1980 to 1993. Ann Intern Med 1996; 124: 407-413.

9 Desai SR, Nicholson AG, Stewart S, et al. Benign pulmonary lymphocytic infiltration and amyloidosis: computed tomographic and pathologic features in three cases. J Thorax Imaging 1997; 12: 215-220.

10 Kobayashi H, Matsuoka R, Kitamura S, Tsunoda N, Saito K. Sjogren's syndrome with multiple bullae and pulmonary nodular amyloidosis. Chest 1988; 94: 438-440.

11 Jeong YJ, Lee KS, Chung MP, et al. Amyloidosis and lymphoproliferative disease in Sjogren syndrome: thinsection computed tomography findings and histopathologic comparisons. J Comput Assist Tomogr 2004; 28: 776-781.

12 Ishibashi $H$, Akamatsu $H$, Sunamori $M$, Ishibashi $T$, Iwata T. Nodular pulmonary amyloidosis with bullae: report of a case. Kyobu Geka 2002; 55: 1069-1072.

13 Ohdama S, Akagawa S, Matsubara O, Yoshizawa Y. Primary diffuse alveolar septal amyloidosis with multiple cysts and calcification. Eur Respir J 1996; 9: 1569-1571.
14 Vitali C, Bombardieri S, Jonsson R, et al. Classification criteria for Sjogren's syndrome: a revised version of the European criteria proposed by the American-European Consensus Group. Ann Rheum Dis 2002; 61: 554-558.

15 Abe Y, Utsunomiya H, Tsutsumi Y. Atypical carcinoid tumour of the lung with amyloid stroma. Acta Pathol Jpn 1992; 42: 286-292.

16 Richmond I, Hasleton PS, Samadian S. Systemic amyloid associated with carcinoma of the bronchus. Thorax 1990; 45: 156-157.

17 Benharroch D, Sukenik S, Sacks M. Bronchioloalveolar carcinoma and generalized amyloidosis complicating progressive systemic sclerosis. Hum Pathol 1992; 23: 839-841.

18 Vanatta PR, Silva FG, Taylor WE, Costa JC. Renal cell carcinoma and systemic amyloidosis: demonstration of AA protein and review of the literature. Hum Pathol 1983; 14: 195-201.

19 Khoor A, Myers JL, Tazelaar HD, Kurtin PJ. Amyloid-like pulmonary nodules, including localized light-chain deposition: clinicopathologic analysis of three cases. Am J Clin Pathol 2004; 121: 200-204. 